\title{
„Es gibt gute Menschen, die hier leben, und es gibt schlechte Menschen"1. Sprachliche Verfahren der Konstitution von Diskursgemeinschaften am Beispiel des Migrationsdiskurses
}

\author{
„Es gibt gute Menschen, die hier leben, und es gibt schlechte Menschen“ - \\ Linguistic Practices for the Constitution of Discourse Communities using \\ the Example of the Migration Discourse
}

\begin{abstract}
ZUSAMMENFASSUNG
Politische Kommunikation zielt häufig auf die Etablierung und Fundierung von Gemeinschaft. Durch die Teilnahme am Diskurs positionieren sich die Diskursakteur*innen nicht nur zur gegebenen Thematik, sondern auch zu anderen Teilnehmer*innen am Diskurs; sie sind dabei häufig Akteur*innen und/oder Repräsentant*innen spezifischer communities of practice (vgl. dazu Eckert \& McConnelGinet, 1992). Ausgehend von diesen Überlegungen untersucht der Beitrag sprachliche Handlungen mit gemeinschaftsstiftender oder -verweigernder Wirkung anhand eines Korpus aus Plenardebatten innerhalb des bundesdeutschen Migrationsdiskurses. Im Fokus der diskursanalytischen Untersuchung steht die sprachliche Realisierung von Selbst- und Fremdpositionierungen.

Schlüsselwörter: Migration, politischer Sprachgebrauch, Diskursgemeinschaften, Positionierung, Stance
\end{abstract}

\section{ABSTRACT}

Political communication often aims at establishing a community. By participating in a discourse, the discourse actors position themselves not only on the given topic, but also with other participants in the discourse; they are often actors and/or representatives of specific communities of practice (cf. Eckert \& McConnel-Ginet 1992). Based on these considerations, the contribution examines linguistic practices with an effect of creating or denying community at the example of the German migration discourse. Applying a discourse analytical approach, the analysis of a corpus of plenary debates focuses on the linguistic realisation of self- and other-positioning. Keywords: migration, political language use, discourse communities, positioning, stance

1 DB 19/138 (BÜNDNIS 90/DIE GRÜNEN mit Bezug auf den Sprachgebrauch der AfD)

Hanna Völker, Institut für Germanistische Sprachwissenschaft, AG Pragmalinguistik, Philipps-Universität Marburg, Pilgrimstein 16, 35032 Marburg, Phone: 004964212824678, hanna.voelker@uni-marburg.de, https://orcid.org/0000-0002-1452-8864

Constanze Spieß, Institut für Germanistische Sprachwissenschaft, AG Pragmalinguistik, Philipps-Universität Marburg, Pilgrimstein 16, 35032 Marburg, constanze.spiess@uni-marburg. https://orcid.org/ 0000-0002-2120-6804 


\section{Einleitung}

Zuwanderung und Migration hat es - so Mecheril (2012, S. 15) - „zu allen historischen Zeiten und fast überall gegeben“. Für Deutschland lassen sich seit dem Ende des Zweiten Weltkriegs unterschiedliche Phasen und Formen von Migration feststellen. Die unmittelbare Nachkriegszeit war geprägt von Debatten um Flüchtlinge und displaced persons, woran ab Anfang der 1960er Jahre die Debatten um Ostzonen- bzw. DDR-Flüchtlinge anschlossen. Es folgten die Phase des Sprechens über Gastarbeiter ab Mitte der 1960er Jahre sowie in den 1970er und 1980er Jahren die Debatten um Asylbewerber und Asylanten (vgl. Niehr, 2020, S. 225). Die aktuelle bundesdeutsche Migrationsdebatte, die infolge der weltweit zunehmenden Migrationsbewegungen ab 2014 bzw. 2015 unter dem Schlagwort Flüchtlingskrise geführt wird, reiht sich in diesen historischen Abriss ein. Von linguistischer Seite liegen zum Migrationsdiskurs der Nachkriegszeit eine Reihe von Arbeiten vor, weshalb er als ein besonders gründlich erforschter öffentlicher Diskurs gelten kann (vgl. Niehr, 2020, S. 225 sowie Spieß, 2019, S. 5)².

Sprachliche Auseinandersetzungen um Migration und Zuwanderung sind seit dem Ende des Zweiten Weltkriegs feste Bestandteile öffentlich-politischer und demokratischer Debattenkultur und damit haben sie auch erheblichen Anteil an Meinungsbildungsprozessen (vgl. Niehr, 2004).

Öffentlich-politische Diskurse um Migration sind demzufolge immer schon damit verbunden, dass durch sprachliches Handeln soziale Gruppen konstituiert werden. Die sprachliche Konstitution sozialer Gruppen geschieht durch unterschiedliche sprachliche Handlungen, u.a. durch Abgrenzungs- und Bewertungshandlungen. Abgrenzungs -und Bewertungshandlungen werden über das Konzept der Positionierung und des Stancetaking linguistisch greifbar.

\section{Theorie: die Konzepte der Positionierung und des Stancetaking}

Das der Untersuchung zugrundeliegende Korpus von parlamentarischen Debattenreden ist dadurch gekennzeichnet, dass in den Reden sehr deutlich Meinungen konstituiert, wiedergeben, kommentiert und dementsprechend Bewertungsaktivitäten vorgenommen werden, was u.a. durch die Handlung des Argumentierens realisiert wird. D.h. die politischen Akteur*innen der Debattenreden positionieren sich argumentativ zum Sachverhalt und beziehen durch das Vorbringen von Argumenten/Prämissen Stellung. Die Bewertungsaktivitäten schlagen sich aber auch

2 So sind aus der Düsseldorfer Schule zahlreiche diskurshistorische Studien hervorgegangen (u.a. Jung, Niehr, \& Böke, 2000; Jung, Wengeler, \& Böke, 1997; Niehr, 2004; Wengeler, 2003); kritisch-diskurslinguistische Arbeiten liegen u.a. vor bei Jäger und Januschek (1992), Reisigl (2007), Reisigl und Wodak (2001), Wodak (2016). 
(unabhängig von der Argumentation als solcher) in der Verwendung wertender Lexik nieder, die mit argumentativen Kontexten verknüpft ist ${ }^{3}$.

Wie ein solches Positionieren und Stellung beziehen theoretisch beschrieben werden kann, haben Lucius-Hoene und Deppermann (2004) und Deppermann (2015) im Hinblick auf das Positionieren in der Face-to-Face-Kommunikation erläutert. Du Bois (2007), Englebretson (2007) oder Jaffe (2009) beziehen das Positionieren als Teil von Stancetakingaktivitäten ebenfalls auf prototypische Faceto-Face-Interaktionen und damit ebenso auf gesprochene Sprache. Die Konzepte sind aber durchaus auch erweiterbar und auf medial wie konzeptionell schriftliche Kommunikation anwendbar (vgl. hierzu Spieß, 2018a), was im vorliegenden Beitrag durch den Bezug auf politische Debattenreden gezeigt wird ${ }^{4}$.

Du Bois (2007) sieht die Aktivität des Positionierens innerhalb von Stancetakingaktivitäten als Teil derselben verortet. Stancetaking und Positioning bzw. Positionierung sind diskursive Aktivitäten, die sprachliche Handlungen konstituieren und die durch sprachliche Handlungen zur Geltung kommen. Sie sind Elemente jeglicher Kommunikation, da sprachliches Handeln immer schon aus einer bestimmten Perspektive erfolgt, so dass mehr oder weniger stark in den verschiedensten kommunikativen Praktiken Sachverhalte durch Stellung beziehende Akte bewertet werden. Du Bois (2007) beschreibt den Akt des Stancetaking, als ,[to] evaluate something, and thereby position myself, and thereby align with you" (S. 163). Demnach umfassen Akte des Stancetaking drei wesentliche Aspekte:

- den Aspekt der Bewertung von Sachverhalten, Gegenständen, Objekten, Ideen, Handlungen usw.;

- den Aspekt der Positionierung der Akteur*innen zum Sachverhalt, Gegenstand, Objekt, zur Idee bzw. Handlung usw.; und zugleich

- die Ausrichtung der Akteur*innen untereinander.

Die Ausrichtung der Akteur*innen untereinander kann dabei explizit oder implizit erfolgen. Implizit zeigt sich diese Ausrichtung beispielsweise durch die Verwendung von sprachlichen Phänomenen, die nicht mit der je anderen Position/ Gruppe in Verbindung gebracht wird und sich auf der lexikalischen Ebene beispielsweise im Nominationskonzept theoretisch fassen lässt.

3 Die Abwertung von Personen durch sprachliche Diskriminierungshandlungen auf lexikalischer Ebene exemplifiziert das folgende Beispiel, in dem die durch die dreifache Iteration gekennzeichnete argumentative Forderung bereits durch die Aneinanderreihung von Personenbezeichnungen mit spezifischen evaluativen Bedeutungsaspekten vorbereitet wird: „Aber die Glücksritter, die Ausreisepflichtigen, die IS-Kämpfer und die Sozialhilfetouristen, für die gibt es eine Lösung, und das ist: abschieben, abschieben, abschieben“ (DB 19/101, AfD); vgl. weiterhin die Ausführungen zu Beleg (6) in diesem Beitrag.

4 Prototypische Debattenreden können als konzeptionell schriftlich und medial mündlich realisiert betrachtet werden. 
Englebretson (2007) betont, dass Stancetakingakte verschiedene sprachliche Ebenen betreffen, insofern er diese Akte im Anschluss an Finegan und Biber (1989, S. 92) als ,the lexical and grammatical expression of attitudes, feelings, judgements, or commitment concerning the propositional content of a message" definiert. (Englebretson, 2007, S. 17). Er kategorisiert Stance als „Form-Meaning relationship"(S. 17), wobei Bedeutung hier in einem gebrauchstheoretischen Sinn aufgefasst werden muss, da es sich um sprachliche Handlungen handelt, die bestimmte Funktionen innehaben. Der Akt der Positionierung als Teil des Stancetakings kann mit Deppermann (2015) und Lucius-Hoene und Deppermann (2004) konkretisiert und auf jegliche Kommunikationssituationen angewandt werden.

Positionierung bezeichnet zunächst ganz allgemein die diskursiven Praktiken, mit denen Menschen sich selbst- und andere in sprachlichen Interaktionen aufeinander bezogen als Personen her- und darstellen, welche Attribute, Rollen, Eigenschaften und Motive sie mit ihren Handlungen in Anspruch nehmen und zuschreiben, die ihrerseits funktional für die lokale Identitätsherund -darstellung im Gespräch sind [...] (Lucius-Hoene \& Deppermann, 2004, S. 168).

Positionierungen im Kontext von Stancetakingaktivitäten stellen eine ideologisch gebundene Aktivität der Zuschreibung von Attributen, Eigenschaften, Verhaltensweisen als Formen der Bewertung von Sachverhalten dar.

Bei Stancetakingaktivitäten nehmen die Akteur*innen durch sprachliche Mittel Bezug aufeinander, insofern sie sich durch den Sprachgebrauch entweder abgrenzen oder bestimmte Ausdrucksphänomene affirmativ verwenden. Die Akteur*innen bewerten mittels Sprachgebrauch einen Sachverhalt/eine Idee/ein Objekt/eine soziale Gruppe/eine Person etc. und positionieren sich mit dem Sprachgebrauch zu den jeweiligen Akteur*innen. Dabei werden sowohl durch Abgrenzungs- als auch durch Affirmationshandlungen zugleich soziale Gruppen hervorgebracht.

Es lässt sich also festhalten, dass Stancetaking- und Positionierungsaktivitäten von Akteur*innen in Diskursen realisiert werden. Sie sind eingebunden in kommunikative Praktiken sowie situierte Kontexte und bringen zugleich Kontexte hervor. Entgegen der Auffassung sozialpsychologischer Einstellungskonzepte sind Stancetaking- und Positionierungsaktivitäten als dynamische, interaktive Formen der Einstellungs- und Bewertungsbekundung aufzufassen. Im vorliegenden Untersuchungskorpus werden Bewertungshandlungen und Einstellungsbekundungen gegenüber Sprache, Sprachgebrauch und sozialen Gruppen auf unterschiedlichen sprachlichen Ebenen vorgenommen. Positionierungs- und Stancetakingaktivitäten können sich dementsprechend auf allen sprachstrukturellen Ebenen niederschlagen. Sie zeigen sich in der Verwendung wertender Lexik in spezifischen sprachlichen Handlungen und Handlungsmustern und sie dienen letztlich der Erzeugung sozialer Gruppen (vgl. hierzu auch Spitzmüller, 2013).

Im Fokus der Ergebnispräsentation wird nach Beschreibung des Korpus die lexikalische Ebene der Nomination und die Ebene der Argumentationsmuster stehen, die aus einer funktionalen, handlungsorientierten Perspektive beschrieben werden. 


\section{Methode: Handlungsanalyse}

Entsprechend dem diskurslinguistischen Mehrebenenmodell nach Spieß (2011) wird angenommen, dass sich diskursive Praktiken als soziale Handlungen beschreiben lassen, die in ihrer Funktionalität, Situationalität und Kontextspezifik sowie der Thematizität und sprachlichen Struktur zu erfassen sind. Zentrale Untersuchungsebenen diskursiver Handlungen sind die lexematische sowie die größerer Äußerungseinheiten, weiterhin die Text- bzw. Gattungs- und Argumentations-/Topoi-Ebenen. Die zuvor beschriebenen Verfahren des Stancetaking und der Positionierung stellen sich somit als sprachliche Handlungen innerhalb des untersuchten Diskursausschnittes dar, die nachfolgend in ihrer spezifischen funktionalen Bedeutung beschrieben werden.

\section{Korpus}

Die Datengrundlage für die qualitative Untersuchung sprachlicher Verfahren der Konstitution von Diskursgemeinschaften (am Beispiel des Migrationsdiskurses) bilden die stenografischen Protokolle aller thematisch relevanten, i.e. dem Migrationsdiskurs zugehörigen, Bundestagsdebatten im Zeitraum zwischen Mai 2019 und April 2020. Das Korpus umfasst neun (auf der Webseite des Bundestages frei verfügbare) Protokolle; je Protokoll sind bis zu sieben Tagesordnungspunkte mit Verhandlungen aus dem Kontext Migration/Einwanderung möglich (vgl. Tab. 1). Das Korpus umfasst 124.056 Tokens. Die Protokolle sind offizielle und institutionell geprägte Dokumente, die die sprachlichen, zum Teil auch nonverbalen Elemente umfassen, um die Inhalte der Debatten öffentlich zugänglich zu machen (vgl. Schwarze \& Stopfner, 2019, S. 1074). Nach Herunterladen aller als relevant eingestuften Protokolle erfolgte die Auswertung durch Annotation und anschließende Kategorisierung der vorkommenden sprachlichen Äußerungen mit der Funktion bzw. Wirkung der Konstitution von Diskursgemeinschaften.

In Bezug auf die Textsorte ist festzuhalten, dass Beiträge von Politiker*innen in Plenardebatten mehrfachadressiert sind (vgl. Kühn, 1995), da sie nicht nur die unmittelbar an der Debatte beteiligten Kolleg*innen der eigenen Partei und politischen Gegner*innen adressieren, sondern ebenso an anwesende Gäste und durch die mediale Verbreitung (v.a. in Form von Videomitschnitten) insbesondere an eine große Gruppe weiterer Adressat*innen gerichtet sind. Primäre Sprechhandlungen dieser „trialogischen“ (vgl. Dieckmann, 1981, S. 218-220), vorrangig in die Öffentlichkeit gerichteten Kommunikationsform ${ }^{5}$ sind dabei INFORMIEREN sowie in persuasiver Absicht Überzeugen/ÜBerreden, Beeinflussen, Verschleiern (vgl. Burkhardt, 2017, S. 514) und KASCHIEREN (vgl. Klein, 1998). Zusätzlich ist festzuhalten, dass auch sprachliche Handlungen, die das eigene Handeln und die

Mit Dieckmann (1981) kann hier auch von „inszenierter Kommunikation“ gesprochen werden (S. 255-279). 
eigenen Überzeugungen legitimieren, wichtige Sprechhandlungen öffentlich-politischer Kommunikation darstellen (zum Legitimieren vgl. u.a. Girnth \& Hofmann, 2016, S. 14) ${ }^{6}$.

Tabelle 1. Korpuszusammensetzung

\begin{tabular}{|c|c|c|c|}
\hline Plenarprotokoll-Nr & Datum & $\begin{array}{c}\text { Tagesordnungspunkt/ } \\
\text { Zusatztagesordnungspunkt und Thema }\end{array}$ & Tokens \\
\hline 19/98 & 9.5 .2019 & $\begin{array}{c}\text { TOP 3, ZP 3, } 4 \\
\text { Fachkräfteeinwanderungsgesetz } \\
\text { TOP } 12 \text { Gesetz zur Entfristung des } \\
\text { Integrationsgesetzes } \\
\text { TOP } 17 \text { Kommunale Initiativen zur } \\
\text { Flüchtlingsaufnahme }\end{array}$ & 23.378 \\
\hline $19 / 101$ & 16.5.2019 & $\begin{array}{c}\text { TOP } 6 \text { Durchsetzung der Ausreisepflicht } \\
\text { TOP } 17 \\
\text { Ausländerbeschäftigungsförderungsgesetz } \\
\text { TOP } 15 \text { Änderung des } \\
\text { Asylbewerberleistungsgesetzes }\end{array}$ & 13.294 \\
\hline $19 / 105$ & 07.6 .2019 & \begin{tabular}{|c|} 
ZP 12-14 Asyl- und Aufenthaltsrecht \\
ZP 15, ZP 16 Fachkräfteeinwanderungsgesetz \\
ZP 18, ZP 19 \\
Ausländerbeschäftigungsförderungsgesetz \\
\end{tabular} & 29.676 \\
\hline $19 / 119$ & 18.10.2019 & TOP 32 Änderung des Aufenthaltsgesetzes & 6.078 \\
\hline $19 / 138$ & 20.12 .2019 & $\begin{array}{l}\text { TOP } 22 \text { Wohnungsnot und Obdachlosigkeit } \\
\text { ZP } 23 \text { Aktuelle Stunde - Globales } \\
\text { Flüchtlingsforum und Grundrechtekatalog }\end{array}$ & 21.477 \\
\hline $19 / 140$ & 16.1.2020 & $\begin{array}{c}\text { TOP } 8 \text { Finanzielle Lasten der } \\
\text { Migrationspolitik } \\
\end{array}$ & 9.942 \\
\hline $19 / 142$ & 29.1 .2020 & $\begin{array}{c}\text { ZP 2, } 3 \text { Aufnahme unbegleiteter } \\
\text { Flüchtlingskinder }\end{array}$ & 6.828 \\
\hline $19 / 148$ & 4.3.2020 & $\begin{array}{c}\text { TOP } 31 \text { Aufnahmebereitschaft von Städten } \\
\text { und Kommunen }\end{array}$ & 7.509 \\
\hline \multirow[t]{2}{*}{$19 / 155$} & 22.4 .2020 & TOP 6 Europäische Flüchtlingspolitik & 5.874 \\
\hline & & & Total: 124.056 \\
\hline
\end{tabular}

\section{Analyse}

Die Erzeugung von Gemeinschaft erfolgt immer auch durch Abgrenzung von Anderen. Durch ihre sprachlichen Äußerungen im Diskurs positionieren sich die Teilnehmenden nicht nur zum Sachverhalt, sondern auch zu anderen Personen

6 Holly (2012) beschreibt die Sprachhandlungen LEGITIMIEREN und WERBEN als „die eigentlich angestrebten Muster", die sich aufgrund der Inszeniertheit politischer Kommunikation hinter positiv bewerteten Handlungen wie INFORMIEREN und DISKUTIEREN verbergen (S. 4). 
und als Mitglied EINER Gemeinschaft, die sich nach außen abgrenzt. Diese soziale Positionierung stellt nach Spitzmüller eine wichtige diskurslinguistische Funktion dar und bildet ,die Grundlage für Prozesse sozialer Stratifizierung bzw. der Aushandlung und Verfestigung von gesellschaftlichen Hierarchien (Dynamiken der Macht)“ [Hervorhebung original] (Spitzmüller, 2013, S. 268). Im Korpus zeigt sich, dass sich die Debattenteilnehmenden einerseits zueinander ausrichten (i.e. die Parteien untereinander, etwa demokratophile Parteien vs. AfD), und dass sie sich andererseits zum Gegenstand der Debatte (Migration) und seinen Akteur*innen positionieren (Du Bois, 2007, S. 163). Dabei kennzeichnet diese Positionierung die Etablierung einer als homogen verstandenen WIR-Gruppe als Parteien-WIR oder Deutschen-WIR und einer Gruppe der ANDEREN mit Bezug auf Migrant*innen oder gegnerische Parteien (vgl. Pelinka \& Wodak, 2002; Spieß, 2018, S. 42).

\subsection{Etablierung einer Eigengruppe}

Durch die sozialverortenden Illokutionen ZugEHÖRIGKEITEN UND NICHTZUGEHÖRIGkeiten festlegen und Eigenraum und Fremdraum absteCKen, eingrenzen, BeanSPRUCHEN sowie durch Pronominalisierung wird über die Differenzbetonung zu einer anderen Gemeinschaft die sprachliche Handlung GemeInSCHAFT EINER EIGENGRUPPE BILDEN vollzogen (vgl. Lobenstein-Reichmann, 2017, S. 824). Diese Eigengruppe ist innerhalb des Migrationsdiskurses häufig nationalitätsbezogen oder territorial geprägt ${ }^{7}$. Kontextspezifisch kann sie etwa ,Menschen mit deutscher Staatsbürgerschaft‘ (Beleg 2) oder ein Parteien-WIR (Beleg 1) umfassen.

(1)

(SPD): Für uns Sozialdemokratinnen und Sozialdemokraten ist klar: Wir arbeiten dafür, dass alle Menschen einen sicheren Aufenthalt, einen sicheren Stand im Leben haben (Deutscher Bundestag, im Folgenden kurz: DB 19/119) ${ }^{8}$.

(2)

(SPD): Noch immer leben Menschen mit ungesichertem Aufenthalt bei uns. [...] Sie können nicht mehr zurück, aber sie können bei uns auch nicht richtig ankommen (DB 19/119).

Die Eigengruppe wird sich ihrer in den meisten Fällen durch die Abgrenzung von Anderen/Fremden gewahr (durch die sie sie als solche erst erschafft) ${ }^{9}$. Deutlich wird gerade auch im Migrationsdiskurs die topografische Dimension der sprach-

7 In Migrationsdebatten ist oftmals auch der Aspekt einer „Wertegemeinschaft“" unter Hervorhebung distinkter und zu sozialer Unruhe führender Unterschiede kultureller Wertvorstellungen Merkmal verbaler Abgrenzungsmechanismen.

8 Hier wie in den folgenden Korpusbelegen Hervorhebungen durch die Verfasserinnen.

9 Zur wechselseitigen Ermöglichungsbedingung von Eigenem und Fremdem über eine „,negation of a given order" (Laclau \& Mouffe, 1985, S. 126) im hegemonialen Diskurs bei Laclau und Derrida vgl. auch Reckwitz, 2006, S. 344-345; vgl. außerdem Lobenstein-Reichmann, 2017). 
lichen Äußerungen mit gemeinschaftsstiftender oder -abwehrender Funktion. Die statische, verwurzelte und verortete Eigengruppe wird zu den hier untersuchten Debatten (und den darin geforderten sozialpolitischen Maßnahmen) erst durch die die Staatsgrenzen transzendierenden Bewegungen einer nomadenhaften anderen Gemeinschaft veranlasst (vgl. die Konstruktion des Fremden über die Differenz sesshaft - beweglich bei Lobenstein-Reichmann, 2017, S. 830). Die Dichotomie des WIR-SIE gehört zu den einfachsten und populärsten sprachlichen Mitteln, mit denen Gemeinschaft erzeugt werden kann. Die folgende Analyse soll beleuchten, auf welche Arten eine Fremdgruppe konstruiert und prädiziert werden kann.

\subsection{Konstruktion (und Prädikation) einer Fremdgruppe}

Nach Harré und van Langenhove (1991) kann zwischen Selbst- und Fremdpositionierung unterschieden werden. Die diskursive Praxis des "Self and other positioning" beinhalte, dass "within a conversation each of the participants always positions the other while simultaneously positioning him or herself" (S. 398). Im Korpus treten die fremdpositionierten „Anderen“ als passive Objekte, nicht Akteur*innen des Diskurses auf. Im Bundestag wird über diese Anderen verhandelt, je nach (parteispezifischem) Argumentationsziel werden Bilder einer schutzbedürftigen oder gefährlichen Menschengruppe evoziert. Gemäß der politischen Ausrichtung erfolgt über Attribution/Prädikation so die Konstitution einer Gemeinschaft von Hilfsbedürftigen oder von Menschen mit kalkuliert verbrecherischen Absichten (jeweils mit entsprechender deontischer Bedeutung).

\subsection{1 „[E]ine der schwächsten Gruppen im Land“"10}

So wird im Rahmen einer sich für die weitere Aufnahme von Menschen aussprechenden Argumentation das Bild einer schutzbedürftigen Gruppe erzeugt, wie die Korpusbelege 3-5 zeigen. Die Konstitution einer Gruppe der Schutzbedürftigen ist oftmals eingebettet in einen impliziten Humanitäts- ${ }^{11}$, und Solidaritäts-Topos ${ }^{12}$ welche auf die Notwendigkeit entsprechender politischer Maßnahmen verweisen ${ }^{13}$.

${ }^{10}$ DB 19/119 (SPD)

${ }^{11}$ Dem Humanitäts-Topos liegt folgendes Muster zugrunde: WeIl eine EnTscheidung/HandLUNG OdER DEREN Folgen Mit den MENSCHENRECHTEN ÜBEREINSTIMMEN/IHNEN ENTGEGENSTEHEN BZW. AUS HUMANITÄREN ÜBERLEGUNGEN GEBOTEN/ABZULEHNEN SIND, IST DIE ENTSCHEIDUNG/HANDLUNG ZU BEFÜRWORTEN/ABZULEHNEN BZW. AUSZUFÜHREN/NICHT AUSZUFÜHREN (vgl. Wengeler, 2003, S. 310).

12 Der Solidaritäts-Topos kann mit folgender Formel wiedergegeben werden: WeIL LäNDER TeIL einer grösseren Gemeinschaft, Teil eines StaAtenbundes sind, SOlLten SIE bei supranationalen FraGEN GEMEINSAME LÖSUNGEN FINDEN, ZUSAMMENHALTEN (SICH SOLIDARISCH VERHALTEN) UND SICH BEI DER BEWÄLTIGUNG POLITISCHER/WIRTSCHAFTLICHER/SOZIALER AufGaBEN GEGENSEITIG UNTERSTÜTZEN (vgl. Völker, 2017, S. 108).

${ }_{13}$ Wengeler (2003) folgend und im Anschluss an (u.a.) Kienpointner (1992), liegen mit Topoi diskursspezifische Argumentationsmuster vor, die auf komplexen argumentativen Diskurshandlungen basieren und durch u.a. Schlüsselwörter und Metaphern konstituiert werden (vgl. Spieß, 2018b, S. 358). 
(3)

(SPD): Wir haben die Begrifflichkeit wieder gehört: „Vorzugsausländer“, von einem „Ansiedlungsprogramm“ war die Rede. Es geht Ihnen einmal mehr darum, Geflüchtete so darzustellen, als würden sie bevorzugt behandelt, als würde ihnen etwas gegeben und anderen nicht. Sie schüren damit ausgerechnet den Neid auf eine der schwächsten Gruppen im Land, auf Menschen, die Schlimmes erlebt haben, die ihre Heimat verlassen mussten, die um ihre Angehörigen bangen (DB 19/119).

(4)

(SPD): Mitten in Europa - und man kann das gar nicht oft genug betonen - hausen Kinder alleine und schutzlos unter Planen oder in einfachsten Zelten ohne geregelte medizinische Versorgung. Ärzte vor Ort berichten, die Verhältnisse seien zum Teil schlimmer als in den Krisengebieten Afrikas, in denen sie zuvor gewesen seien. [...] Den Kindern fehlt es am Nötigsten, an Schutz und Fürsorge. Es sind die Schwächsten, meine Damen und Herren, die weder etwas für die Konflikte dieser Welt können noch für die Entscheidungen ihrer Eltern. [...] Sie brauchen diesen Schutz; sie brauchen ihn nötiger als alle anderen (DB 19/148).

(5)

(BÜNDNIS 90/DIE GRÜNEN): Wir dürfen aber trotz und gerade angesichts der Coronapandemie die Schwächsten nicht vergessen: weder die Menschen, die weiter vor dem Bürgerkrieg in Libyen über das Mittelmeer fliehen, noch die Menschen, die auf europäischem Boden unter katastrophalen Bedingungen leben müssen und diesem Virus nahezu schutzlos ausgeliefert sind. Es ist unsere Pflicht, dort zu helfen, wo wir es können, dort miteinander solidarisch zu sein, wo wir es können (DB 19/155).

In den Belegen erfolgt die Kontextualisierung einer Gruppe von Schwachen/ Schutzbedürftigen im Rahmen eines Schutz-Topos, der wie folgt expliziert werden kann: Weil die in europäischen Nachbarstaaten angekommenen Menschen auch Dort noch keinen hinReichenden SchutZ erhalten haben, sollte DeutschLAND besonders SChutzbedÜrftige aufnehmen bZw. Massnahmen Verfolgen, die Situation (Der Menschen) zu verbessern. Die Differenzierung zwischen WIRGruppe und Gruppe der ANDEREN erfolgt auf Basis der Kategorien der den Schutz Bedürfender und der den Schutz (aufgrund vorteilhafterer politischer und wirtschaftlicher Verhältnisse) bieten Könnender (vgl. „Es ist unsere Pflicht, dort zu helfen, wo wir es können [...].“, Beleg 5).

\subsection{2 „Die Masse der Illegalen in Deutschland“14}

Von migrationskritischer Seite wird eine große und gefährliche Gemeinschaft benannt und insofern konstruiert. Die Bildung einer WIR-Gruppe und einer Gruppe der ANDEREN/Fremdgruppe erfolgt dabei anhand der diskursiven Erzeugung von Bedrohungsszenarien und Konfliktträchtigem zwischen den Gruppen.

(6)

(AfD): Über diese Naivität lachen sich Hunderttausende Asyltäuscher in aller Welt kaputt (DB 19/105).

${ }^{14}$ DB 19/105 (AfD) 
Über die Strategien der Nomination und Prädikation wird eine Gruppe Hunderttausende[r] Asyltäuscher sprachlich erzeugt, deren Handeln den Interessen der WIR-Gruppe entgegengesetzt wird (Beleg 6). Die realisierte, pauschale Handlungszuschreibung, die in der Personenbezeichnung Asyltäuscher enthalten ist, dass Menschen in aller Welt nämlich nach Deutschland kämen, um sich durch Betrug finanziell zu bereichern, vollzieht sprachliche Diskriminierungs- und Abgrenzungsakte der so konstruierten In- von der Outgroup.

(7)

(AfD): Es entstünde eine Art riesige Rolltreppe, ein endloses Fließband für Migranten aus Orient und Afrika direkt hinein ins Herz unserer Städte und Gemeinden (DB 19/98).

In Beleg (7) wird die potentielle Wirkung einer kommunalen Personenaufnahme als bedrohliche Zukunftsvision im Konjunktiv skizziert, wobei die (aus den Bereichen Wirtschaft und Produktion stammenden) technischen Metaphern der Rolltreppe und des Fließbandes der organischen Metapher Herz gegenübergestellt werden. Die strukturelle Analogie von Migrations- und Fluchtbewegungen mit einer gleichförmigen (und endlosen) Bewegung durch Rolltreppen und Fließbänder wird dabei ebenso präsupponiert wie die Faktizität der skizzierten Folgen der verhandelten politischen Maßnahme. In topografischer bzw. raumsoziologischer Dimension geht mit der Metaphorik die Vorstellung einher, bestimmte politische Maßnahmen könnten einen direkten Einzug der Fremdgruppe in die Mitte der Gesellschaft bewirken und damit eine Ordnung gefährden, die eher marginale Positionen für sie vorsieht. ${ }^{15}$ Die Metaphorik sowie das Quantifizierungsvokabular ordnen die sprachliche Äußerung dabei in exkludierender Richtung ein.

Sprachliche Mittel zur Konstruktion eines Feindbildes für eine migrationsskeptische Argumentation können sich zu Topoi verdichten, die verhandelt, ggfs. explizit zurückgewiesen werden. Beleg (8) stellt einen Redebeitrag dar, in welchem die verbal realisierte Dichotomie und prädizierte Ungleichheit sozialer Gruppen aus einem vorangegangenen Beitrag ausgehandelt wird. Zur Funktion dieser Sprachhandlung gehört dabei auch, die politischen Gegner*innen ob dieser Dichotomisierung zu kritisieren und sich somit zu positionieren.

(8)

(BÜNDNIS 90/DIE GRÜNEN): Damit unterteilen Sie Menschen in unterschiedliche Kategorien. Es gibt gute Menschen, es gibt schlechte Menschen. [...] Der Antrag, den Sie hier gestellt haben, ist schlicht und einfach rassistisch, (Beifall beim BÜNDNIS 90/DIE GRÜNEN und bei der SPD sowie bei Abgeordneten der LINKEN - Widerspruch bei der AfD) weil Sie die Menschen in unterschiedliche Kategorien stecken. Es gibt gute Menschen, die hier leben, und

${ }^{15}$ Zur Topografie des Eigenen und Fremden vgl. Lobenstein-Reichmann (2017) und Waldenfels (1997). 
es gibt schlechte Menschen. Es sind diejenigen aus anderen Ländern, aus dem Süden dieses Kontinents, aus Afrika, Araber, die Sie als Untermenschen kategorisieren, die für Sie weniger wert sind als die Menschen, die hier geboren sind (DB 19/138).

Im Korpus finden sich Belege für die Erzeugung einer Gemeinschaft über Bedrohungsszenarien in den Sprechhandlungen der AfD, während sich die Äußerungen vonseiten der anderen Regierungsparteien klassifizieren lassen in Gruppenkonstitution im Rahmen des Schutz-Topos oder, vor allem bei der FDP, mit Verweis auf eine FACHKRÄFTE-Gruppe innerhalb einer arbeitsmarktpolitisch orientierten Argumentation:

(9)

(FDP): Ja, wir müssen drei Gruppen klar voneinander trennen: Erstens: Wer braucht unseren Schutz? Zweitens: Wen wollen wir auf unseren Arbeitsmarkt und in unsere Gesellschaft einladen? Drittens: Für wen gilt weder noch? (DB 19/105)

(10)

(FDP): Sorgen Sie für eine geregelte Einwanderung nach Deutschland, damit Fachkräfte auch die Chance haben, auf unserem Arbeitsmarkt Fuß zu fassen! (DB 19/119).

\subsection{Konkurrenz der konstruierten Gemeinschaften}

(AfD): Laut türkischer Regierung haben sich bereits mehr als 80000 Migranten auf den Weg gemacht, die griechische Grenze zu stürmen, weitere dreieinhalb Millionen sitzen auf gepackten Koffern, und Experten rechnen damit, dass durch die aktuelle Lage diese Zahl auf bis zu 6 Millionen ansteigt. Das sind weitere 6 Millionen Menschen auf der Suche nach Wohnungen, Schulen und Kitaplätzen. Sie zündeln hier also am sozialen Frieden in unserem Land (DB 19/148).

Das Evozieren eines Bedrohungsszenarios kann dafür genutzt werden, eine Konkurrenz zwischen Gruppen zu markieren, die es, so der implizite Schlussprozess, zu verhindern gelte. Sichtbar wird dies im untersuchten Diskursausschnitt bei dem Subthema Wohnen, das argumentativ mit dem Thema Migration in Verbindung gebracht wird. Der Konkurrenz-Topos (als Variante des Belastungs-Topos) funktioniert nach folgendem Muster: WeIl die Aufnahme von Geflüchteten die WOHNUNGS- UND BILDUNGSPOLITISCH ANGESPANNTE SitUation NOCH WEITER BELASTET/ VERSCHÄRFT, IST SIE ABZULEHNEN UND SIND ENTSPRECHENDE MASSNAHMEN ZU IHRER VerhindERUNG ZU ERGREIFEN. Präsupponiert wird eine direkte Kausalität zwischen Migrationsbewegungen und sozialem Frieden (Beleg 11) und das Bestehen negativer Konkurrenz- und Wettbewerbsbeziehungen zwischen den konstruierten Gruppen. Die Brandstiftermetaphorik (am sozialen Frieden zündeln) unterstützt diese Argumentation, indem anhaltende Migrationsbewegungen als Bedrohung des sozialen Friedens gewertet werden.

Deutlich wird an diesen Beispielen auch, wie sprachliche Handlungen wirklichkeitskonstitutiv wirksam werden. Die Gegenüberstellung und in-Konkurrenz- 
Setzung sozialer Gruppen setzt diese (in ihrem Bestehen) zum einen als Faktizität voraus. Die Konstruktion von Gruppenverhältnissen durch Präsupposition geht in diesem Fall auch einher mit der Schaffung von „Raum-, Besitz- und Zugehörigkeitsverhältnissen“ (Lobenstein-Reichmann, 2017, S. 811), indem die dargestellten sozialpolitischen Verhältnisse als vorsprachlich gegebene vorausgesetzt werden. Die Verbindung eines unkontrollierbaren Bedrohungsszenarios mit der Darstellung einer Konkurrenz zwischen sozialen Gruppen, die als Eigengruppe/ Ingroup und Fremdgruppe/Outgroup markiert werden, kann als exkludierend eingeordnet werden. Damit werden Sprechhandlungen für die gesellschaftliche Wirklichkeit wirksam vgl. Berger und Luckmann (2018).

\section{Schluss}

Ausgehend von linguistischen Konzepten zur Positionierung innerhalb von öffentlichen Diskursen wurden sprachliche Verfahren der Konstituierung von Diskursgemeinschaften unter Bezugnahme auf Diskursfragmente um Migration, Einwanderung und Flucht in Deutschland analysiert. Im Vordergrund der sprachgebrauchstheoretischen Untersuchung standen Praktiken der Nomination und des argumentativen Handelns, die für die diskursive Erzeugung von Eigen- und Fremdgruppen konstitutiv sind sowie im Sinne einer Konkurrenzschaffung der konstruierten Gemeinschaften eingesetzt werden können und die zugleich Formen des Stancetaking darstellen, insofern durch die Ausdrucksverwendungen Einstellungen gegenüber Sachverhalten und sozialen Gruppen zur Geltung gebracht werden. Einerseits können anhand von Pronominalisierung und wertender Lexik inkludierende Sprechakte realisiert werden, andererseits unterstützen diese sprachlichen Praktiken in Verbindung mit Metaphorik innerhalb exkludierender sprachlicher (Argumentations-)Handlungen Bedrohungsszenarien, die ex negativo die Konstitution einer Ingroup verfestigen helfen. In parlamentarischen Debattenreden positionieren sich die Akteur*innen qua Sprachgebrauch nicht nur zum verhandelten Sachverhalt, sondern auch zueinander und vollziehen diskursive Handlungen mit identitätsstiftender oder differenzorientierter Funktion.

\section{References}

Berger, P. L., \& Luckmann, T. (2018). Die gesellschaftliche Konstruktion der Wirklichkeit. Frankfurt am Main: Fischer.

Burkhardt, A. (2017). Plenardebatten. In T. Niehr, J. Kilian, \& M. Wengeler (Eds.), Handbuch Sprache und Politik in 3 Bänden: Vol. 2 (pp. 508-531) Bremen: Hempen.

Deppermann, A. (2015). Positioning. In A. De Fina, \& A. Georgakopoulou (Eds.), Handbook of Narrative Analysis (pp. 369-387). New York: Wiley. DOI: 10.1002/9781118458204.ch19.

Deutscher Bundestag (DB). Stenografischer Bericht. 19. Wahlperiode. Bonn 2019-2020. Retrieved December 7, 2020, from https://www.bundestag.de/dokumente/protokolle/.

Dieckmann, W. (1981). Politische Sprache - Politische Kommunikation. Vorträge-Aufsätze - Entwürfe. Heidelberg: Winter. 
Du Bois, J. (2007). The stance triangle. In R. Englebretson (Ed.), Stancetaking in Discourse. Subjectivity, evaluation, interaction (pp. 139-182). Amsterdam, Philadelphia: John Benjamins. DOI: 10.1075/pbns.164.07du.

Eckert P., \& McConnell-Ginet, S. (1992). Think practically and look locally: language and gender as community-based practice. Annual Review of Anthropology, 21, 461-90. DOI: 10.1146/annurev.an.21.100192.002333.

Englebretson, R. (Ed.). (2007). Stancetaking in Discourse. Subjectivity, evaluation, interaction (= Pragmatics and Beyond, New Series 164). Amsterdam: Benjamins. DOI: 10.1075/pbns.164.

Finegan, E., \& Biber, D. (1989). Styles of stance in English: Lexical and grammatical marking of exidentiality and effect. Text, 9(1), 93-124. DOI: 10.1515/text.1.1989.9.1.93.

Girnth, H., \& Hofmann, A. A. (2016). Politolinguistik. Heidelberg: Winter. DOI: 10.33675/201682537590 .

Harré, R., \& Langenhove, L. van (1991). Varieties of positioning. Journal for the Theory of Social Behaviour, 21(4), 393-407. DOI: 10.1111/j.1468-5914.1991.tb00203.x.

Holly, W. (2012). Sprache und Politik. Pragma- und medienlinguistische Grundlagen und Analysen. Berlin: Frank \& Timme.

Jaffe, A. (2009). Introduction. The Sociolinguistics of Stance. In A. Jaffe (Ed.), Stance. Sociolinguistic Perspectives (pp. 3-28). Oxford: Oxford University Press.

Jäger, S. \& Januschek, F. (Eds.). (1992). Der Diskurs des Rassismus. Ergebnisse des DISS-Kolloquiums November 1991. (Osnabrücker Beiträge zur Sprachtheorie OBST 46). Duisburg: Universitätsverlag Rhein-Ruhr.

Jung, M., Niehr, T., \& Böke, K. (2000). Ausländer und Migranten im Spiegel der Presse. Ein diskurshistorisches Wörterbuch zur Einwanderung seit 1945. Wiesbaden: Westdeutscher Verlag.

Jung, M., Wengeler, M., \& Böke, K. (1997). Die Sprache des Migrationsdiskurses. Das Reden über „Ausländer “ in Medien, Politik und Alltag. Opladen: Westdeutscher Verlag.

Kienpointner, M. (1992). Alltagslogik. Struktur und Funktion von Argumentationsmustern. Stuttgart-Bad Cannstadt: Frommann-Holzboog.

Klein, J. (1998). Politische Kommunikation als Sprachstrategie. In O. Jarren, U. Sarcinelli, \& U. Saxer (Eds.), Politische Kommunikation in der demokratischen Gesellschaft. Ein Handbuch mit Lexikonteil (pp. 376-395). Opladen, Wiesbaden: Westdeutscher Verlag.

Kühn, P. (1995). Mehrfachadressierung. Untersuchungen zur adressatenspezifischen Polyvalenz sprachlichen Handelns. Tübingen: Niemeyer. DOI: 10.1515/9783110926835.

Laclau, E., \& Mouffe, C. (1985). Hegemony and Socialist Strategy. Towards a Radical Democratic Politics (2nd ed.). London, New York: Verso.

Lobenstein-Reichmann, A. (2017). Eigenes und Fremdes konstruieren. In T. Niehr, J. Kilian, \& M. Wengeler (Eds.), Handbuch Sprache und Politik in 3 Bänden: Vol. 2 (pp. 811-832). Bremen: Hempen.

Lucius-Hoene, G., \& Deppermann, A. (2004). Narrative Identität und Positionierung. Gesprächsforschung online, 5, 166-183. Retrieved November 11, 2020, from http://www.gespraechsforschung-ozs.de/fileadmin/dateien/heft2004/ga-lucius.pdf.

Mecheril, P. (2012). Migrationsgesellschaft. In A. Kriwak, \& G. Pallawer (Eds.), Medien und Minderheiten (pp. 15-35). Innsbruck: innsbruck university press.

Niehr, T. (2004). Der Streit um Migration in der Bunderepublik Deutschland, der Schweiz und Österreich. Eine vergleichende diskursgeschichtliche Untersuchung. Heidelberg: Winter.

Niehr, T. (2020). Migrationsdiskurs. In T. Niehr, J. Kilian, \& J. Schiewe (Eds.), Handbuch Sprachkritik (pp. 225-232). Berlin: Metzler. DOI: 10.1007/978-3-476-04852-3_29.

Pelinka, A., \& Wodak, R. (Ed.). (2002). „Dreck am Stecken“. Politik der Ausgrenzung. Wien: Czernin. 
Reckwitz, A. (2006). Ernesto Laclau. Diskurse, Hegemonien, Antagonismen. In S. Moebius, \& D. Quadflie (Eds.), Kultur. Theorien der Gegenwart (pp. 339-349). Wiesbaden: Verlag für Sozialwissenschaften. DOI: 10.1007/978-3-531-90017-9_27.

Reisigl, M. (2007). Nationale Rhetorik in Fest- und Gedenkreden. Eine diskursanalytische Studie zum ,österreichischen Millennium “in den Jahren 1946 und 1996. Tübingen: Stauffenburg.

Reisigl, M. \& Wodak, R. (2001). Discourse and Discrimination. Rhetorics of racism and antisemitism. London, New York: Routledge.

Schwarze, C., \& Stopfner, M. (2019). Die politische Rede in Österreich und der Schweiz. In A. Burkhardt (Ed.), Handbuch Politische Rhetorik (Reihe Handbücher Rhetorik, 10) (pp. 1067-1092). Berlin, Boston: De Gruyter. DOI: 10.1515/9783110331516-048.

Spieß, C. (2011). Diskurshandlungen. Theorie und Methode linguistischer Diskursanalyse am Beispiel der Bioethikdebatte. Berlin, Boston: De Gruyter.

Spieß, C. (2018a). „Deutschland muss Deutschland bleiben“ - Sprachliche Selbst- und Fremdpositionierungsaktivitäten im Kontext politischer Äußerungen über Migration am Beispiel des Ausdrucks 'Leitkultur'. Zeitschrift für Kulturwissenschaft, 1, 35-55. DOI: 10.2478/kwg-20180003.

Spieß, C. (2018b). Diskurs und Handlung. In I. H. Warnke (Ed.), Handbuch Diskurs (pp. 339-362). Berlin, Boston: De Gruyter. DOI: 10.1515/9783110296075-014.

Spieß, C. (2019). „Die Anderen als Gefahr“. Geschichte, Funktion und Bedeutung der Metapher FESTUNG EUROPA im Diskurs um Migration und Zuwanderung 2015-2017. Mythos-Magazin: Politisches Framing 1, 1-15.

Spitzmüller, J. (2013). Metapragmatik, Indexikalität und soziale Registrierung. Zeitschrift für Diskursforschung, 3, 263-287.

Völker, H. (2017). Is the German Immigration Debate Changing? A Discourse Linguistic Approach to the German Immigration Debate. On Changes since the 1970s. 10plus1 Living Linguistics: The Linguistics of Politics, 3, 87-108. Retrieved December 7, 2020, from http://10plus 1journal. com/?page_id=868.

Waldenfels, B. (1997). Topographie des Fremden. Studien zur Phänomenologie des Fremden. Frankfurt am Main: Suhrkamp.

Wengeler, M. (2003). Topos und Diskurs. Begründung einer argumentationsanalytischen Methode und ihre Anwendung auf den Migrationsdiskurs (1960-1985) (Reihe Germanistische Linguistik, 244). Tübingen: Niemeyer. DOI: 10.1515/9783110913187.

Wodak, R. (2016). Politik mit der Angst. Zur Wirkung rechtspopulistischer Diskurse. Wien, Hamburg: Edition Konturen. 